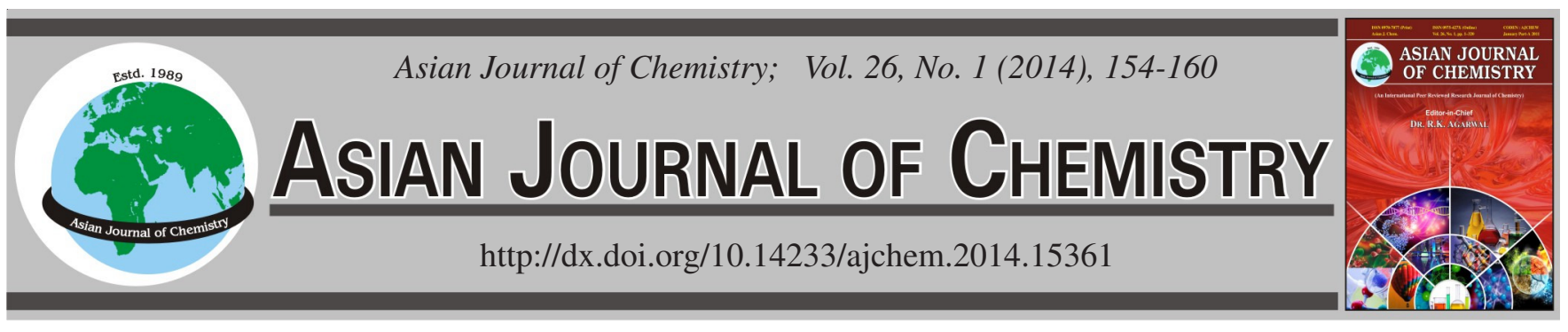

\title{
Synthesis, Characterization and Properties of Hydrophobically Modified Polyacrylamides
}

\author{
W. KUANG ${ }^{1}$, R.Y. $\mathrm{LI}^{2}$, J. ZHANG ${ }^{2}$, B. WU ${ }^{1,3, *}$ and Y.B. TAN ${ }^{1,3, *}$
}

${ }^{1}$ School of Chemistry and Chemical Engineering, Shandong University, Jinan 250100, Shandong Province, P.R. China

${ }^{2}$ Department of Technology Research, CNOOC Research Center, State Key Laboratory of Offshore Oil Exploitation, Beijing 100027, P.R. China ${ }^{3}$ Key Laboratory of Special Functional Aggregated Materials, Shandong University, Ministry of Education, Jinan 250100, P.R. China

*Corresponding authors: Fax: +86 531 88564464; Tel: +86 531 88363502; E-mail: ybtan@ @sdu.edu.cn; wubo@ sdu.edu.cn

Hydrophobically modified polyacrylamides were synthesized by the micellar copolymerization of acrylamide, 3-[N-(2-methacryloxyethyl)$\mathrm{N}, \mathrm{N}$-dimethylamino]propane sulfonate and di- $n$-octylmethacrylamide using 2,2'-azobis(2-methylpropionamidine)dihydrochloride as the initiator. The structure, composition, molecular weight of copolymers were studied via ${ }^{1} \mathrm{H}$ NMR, element analyse and static light scattering. Their thermal properties and aggregation properties of the copolymers were investigated by thermogravimetric analyzer, fluorescence spectroscopy, dynamic light scattering and surface tension. Thermal degradation and viscosity stability showed that hydrophobically modified polyacrylamide can greatly improve salt resistance and thermal stability and it has the high practical application performance in oil recovery.

Keywords: Water-soluble copolymer, Hydrophobic association, Hydrophobically modified polyacrylamide.

\section{INTRODUCTION}

Hydrophobically modified water-soluble polymers are important functional materials that have a wide range of applied prospects, such as oil fields exploiting, water purifying, papermaking, drug delivering and releasing, personal caring, etc. $^{1-3}$. Among the water-soluble polymers hydrophobically modified polyacrylamides (HM-PAM) have attracted increasing attention on a multitude of important biological functions and industrial applications. Although many uses for polymers of this type have been suggested as as aqueous viscosity modifiers in tertiary oil recovery and latex paint systems ${ }^{4-7}$. The commonly used HM-PAM have the incorporation of a relatively low amounts of a hydrophobic comonomer $(\leq 5 \mathrm{~mol} \%)$ into a hydrophilic polyacrylamides backbone results in the formation of systems with unique rheological properties. Due to hydrophobic interactions, these polymers can associate in aqueous solutions with a formation of hydrophobic microdomains. In the past few years, some interesting studies have been reported a series of novel HM-PAM containing a N,N-dialkylacrylamide. Making the comparison for the same total hydrophobe chain length, double-chain hydrophobes considerably enhance the thickening efficiency with respect to single chain hydrophobes ${ }^{8,9}$.

In this article, a micellar synthesis of twin-tailed hydrophobically associating copolymers composed of acrylamide (AM), 3-[N-(2-methacryloxyethyl)-N,N-dimeth-ylammonio]- propane sulfonate (DMAPS) and di- $n$-octylmethacrylamide (DOMAM) is described and the copolymers were characterized by ${ }^{1} \mathrm{H}-\mathrm{NMR}$, elemental analysis and TGA. Then their properties in aqueous solution were studied by dynamic light scattering, surface tension and fluorescence measurements. Besides, the degradation of samples in simulated mineralized water was carried out. The results showed that PADO exhibited better performance of salt tolerance and thermal stability, which indicated that it might have a potential application in oilfield exploitation.

\section{EXPERIMENTAL}

Acrylamide (AM, Tianjin Chemical Reagent Co.) were further purified by three-time recrystallization dried at room temperature. 2,2'-Azobis (2-methylpropionamidine)dihydrochloride (AIBA, Qingdao Run Xing Photoelectric Materials Co.) and sodium dodecyl sulfate (SDS, Shanghai Chemical Reagent Co.) were used as received. Di- $n$-octylamine $(\geq 97 \%$ purity) were purchased from J \& K Chemical. 3-[N-(2methacryloxyethyl)-N,N-dimethylammonio]-propane sulfonate (DMAPS) was prepared according to the literature ${ }^{10,11}$. All other reagents were obtained from commercial sources and were of analytical grade.

Synthesis of twin-tailed monomers: The hydrophobic twin-tailed monomers (DOMAM) were synthesized using a process similar to originally described by McCormick et al. ${ }^{12}$ and others ${ }^{13,14} .10 \mathrm{mmol} \mathrm{L}^{-1}$ di- $n$-octylamine was initially dissolved 
in $100 \mathrm{~mL}$ of methylene chloride along with an equimolar concentration of triethylamine and added into a round bottom flask under cooling with an ice water bath. Methacryloyl chloride $\left(10 \mathrm{mmol} \mathrm{L}^{-1}\right.$ ) was dissolved in $100 \mathrm{~mL}$ methylene chloride and then added dropwise into the flask reaction over a period of at least $3 \mathrm{~h}$ at such a rate under a nitrogen atmosphere. Once the methacryloyl chloride solution was completely incorporated, the mixture was stirred resulting mixture was stirred at a constant temperature $\left(10^{\circ} \mathrm{C}\right)$ for 1 day. Then, triethylamine hydrochloride was removed by filteration and methylene chloride was allowed to evaporate the solvent off under reduced pressure at room temperature. Finally the product (an amber coloured liquid) was puried by distillation with $\mathrm{CuCl}$ as a polymerization inhibitor under vacuum. DOMAM was obtained in $53 \%$ yield. ${ }^{1} \mathrm{H}$ NMR of DOMAM $(400 \mathrm{MHz}$, $\left.\mathrm{D}_{2} \mathrm{O}, \mathrm{ppm}\right): \delta 0.87-0.89\left(6 \mathrm{H}, \mathrm{t},-\mathrm{N}\left(\mathrm{CH}_{2} \mathrm{CH}_{2} \mathrm{CH}_{2} \mathrm{CH}_{2} \mathrm{CH}_{2} \mathrm{CH}_{3}\right)_{2}\right)$;

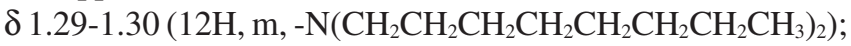
$\delta$ 1.51-1.54(4H,m, $\left.-\mathrm{N}\left(\mathrm{CH}_{2} \mathrm{CH}_{2} \mathrm{CH}_{2} \mathrm{CH}_{2} \mathrm{CH}_{2} \mathrm{CH}_{3}\right)_{2}\right) ; \delta 3.30-3.31$ $\left(4 \mathrm{H}, \mathrm{t},-\mathrm{N}\left(\mathrm{CH}_{2} \mathrm{CH}_{2} \mathrm{CH}_{2} \mathrm{CH}_{2} \mathrm{CH}_{2} \mathrm{CH}_{3}\right)_{2}\right) ; \delta 1.95\left(3 \mathrm{H}, \mathrm{s}, \mathrm{CH}_{3}-\mathrm{C}=\right)$; $\delta$ 4.97,5.10 (2H, d, d, $\left.\mathrm{CH}_{2}=\right)$.

Synthesis of hydrophobically modified polyacrylamides (PADO): All samples were prepared using a micellar copolymerization route ${ }^{15-18}$. Each reaction was conducted in a $250 \mathrm{~mL}$, three-necked, round-bottomed flask equipped with a condenser, magnetic stirrer, thermometer and gas inlet. Before the synthesis of PADO, the reactor, containing designed mixture of sodium dodecyl sulphate (SDS), acrylamide, DMAPS and hydrophobic monomer (DOMAM) in deionized water, was purged with nitrogen for $70 \mathrm{~min}$ to ensure the complete removal of trapped air. The initiators (AIBA) were added into the reactor when the mixture was homogeneous and the reaction was carried out at $56^{\circ} \mathrm{C}$ for $10 \mathrm{~h}$ with purging nitrogen and vigorous stirring. After cooling the final reaction mixture, the aqueous polymer solutions were precipitated by ethanol and washed by ethanol for five times to remove emulsifier and residual monomers, then dried under vacuum at $50{ }^{\circ} \mathrm{C}$ for 4 days. The detailed process was as shown in Fig. 1.

Due to the viscosity of the reaction mixture, especially in the later stage of synthesis, the total concentration of monomers was kept below 3 wt. \%. The syntheses were carried out with a range of hydrophobic twin-tailed monomer from 0.1-1 mol \% and 5 mol \% DMAPS, indicated in Table-1, which summarize details of the copolymers synthesized.

Characterization: Elemental analyses were performed on Elementar Vario E1 III analyzer (German). All ${ }^{1} \mathrm{H}$ NMR experiments were performed on a Bruker AVANCE400 NMR spectrometer. $\mathrm{D}_{2} \mathrm{O}$ was used for field-frequency lock and the observed ${ }^{1} \mathrm{H}$ chemical shifts were reported in parts per million $(\mathrm{ppm})$ relative to an internal standard (TMS, $\delta \mathrm{ppm})$.

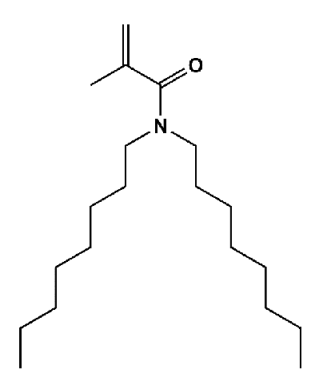

DOMAM

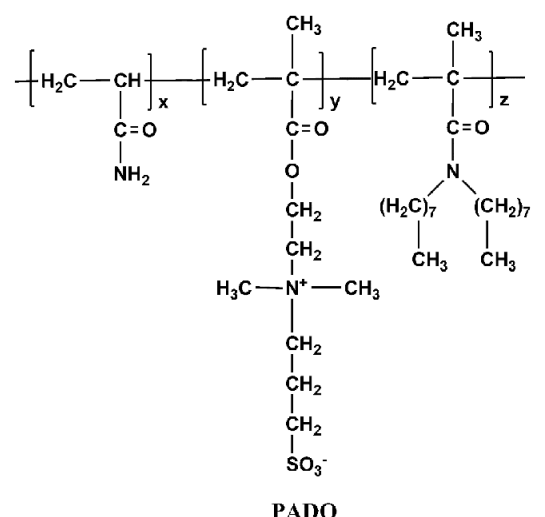

PADO
Fig. 1. Structure of twin-tailed monomers (DOMAM) and copolymer PADO

The weight-average molecular weight $\left(\mathrm{M}_{\mathrm{w}}\right)$ of the samples was determined through static light scattering measurements on a light scattering instrument (Wyatt Technology). The laser is positioned so that the incident beam was vertically polarized. Zimm plots were obtained using Astra software. The copolymers were examined in $0.15 \mathrm{~mol} \mathrm{~L}^{-1} \mathrm{NaCl}$ solution and filtered with a $0.2 \mu \mathrm{m}$ pore size membrane filter prior to static light scattering measurement. Solutions with different concentration were prepared for light scattering measurements by diluting the stock solutions. A differential refractive index detector (Optilab-REX) was used to measure the differential refractive indices $(\mathrm{dn} / \mathrm{dc})$ of different copolymer solutions at $658 \mathrm{~nm}$ and $25^{\circ} \mathrm{C}$.

Thermal characteristics of samples were determined with Thermogravimetric analyzer (TGA). TGA was performed with a Mettler Toledo SDTA-851 TGA system. The analysis was performed with approximately of $10 \mathrm{mg}$ of dried samples in a dynamic nitrogen atmosphere (flow rate $50 \mathrm{~mL} / \mathrm{min}$ ) at a heating rate of $10^{\circ} \mathrm{C} / \mathrm{min}$.

Dynamic light scattering (DLS) measurements were performed on a multiangle laser photometer equipped with a linearly polarized gallium arsenide $(\mathrm{GaAs})$ laser $(\mathrm{k}=658 \mathrm{~nm}$; Wyatt Technology Co. DAWN HELEOS), which were conducted at a scattering angle of $99^{\circ}$. The solution used for light scattering measurements were all filtered through Millipore $0.8 \mu \mathrm{m}$ hydrophilic membranes before using.

Fluorescence spectra were measured with a Hitachi F4500 fluorescence spectrophotometer using $1 \mathrm{~cm}$ quartz cell. Pyrene spectra were recorded with fixed excitation wavelength at 335 $\mathrm{nm}$ and the slit widths of excitation and emission were fixed at 2.5 and $5 \mathrm{~nm}$, respectively. The emission spectra were scanned at the range of $350-500 \mathrm{~nm}$. $\mathrm{I}_{1}$ and $\mathrm{I}_{3}$ which was fluorescence intensity and obtain from the emission intensities at 373 and $384 \mathrm{~nm}$ and the ratio of $\mathrm{I}_{1} / \mathrm{I}_{3}$ was used to estimate the

TABLE-1

FEED RATIO, CONVERSION OF COPOLYMER IN SYNTHESIS AND COMPOSITION OF COPOLYMER DETERMINED via ELEMENTAL ANALYSES

\begin{tabular}{|c|c|c|c|c|c|c|c|}
\hline \multirow{2}{*}{ Sample } & \multirow{2}{*}{$\begin{array}{c}\text { Feed mole ratio of } \\
\text { AM/DMAPS/DOMAM }\end{array}$} & \multirow{2}{*}{$\begin{array}{l}\text { Conv. } \\
\text { (wt. \%) }\end{array}$} & \multicolumn{4}{|c|}{ Elemental analysis (wt \%) } & \multirow{2}{*}{$\begin{array}{c}\text { Actual mole ratio of } \\
\text { AM/DMAPS/DOMAM }\end{array}$} \\
\hline & & & $\mathrm{C}$ & $\mathrm{H}$ & $\mathrm{N}$ & $S$ & \\
\hline PADO-1 & 94.90:5.00:0.10 & 93 & 50.551 & 7.120 & 18.964 & 0.569 & 94.93:4.96:0.11 \\
\hline PADO-2 & 94.70:5.00:0.30 & 88 & 50.610 & 7.132 & 18.941 & 0.562 & 94.78:4.90:0.32 \\
\hline PADO-3 & 94.40:5.00:0.60 & 89 & 50.681 & 7.149 & 18.867 & 0.585 & $94.29: 5.10: 0.61$ \\
\hline PADO-4 & 94.00:5.00:1.00 & 85 & 50.784 & 7.169 & 18.829 & 0.572 & 94.04:4.98:0.98 \\
\hline
\end{tabular}


micropolarity sensed by the pyrene as well as to obtain the critical micelle concentration of the surfactants in aqueous solution at $25^{\circ} \mathrm{C}$. The pyrene concentration was fixed at $1.0 \times$ $10^{-6} \mathrm{~mol} / \mathrm{L}$ in the measurements.

Surface tension measurements were performed on a Kruss K12 Processor Tensiometer equipped with a Wilhelmy plate at $25^{\circ} \mathrm{C}$.

Hydrophobically modified polyacrylamides series ( 0.3 wt. $\%)$ were dissolved, respectively in the simulated water, whose salinity of simulated water was $2743 \mathrm{mg} / \mathrm{L}$ and containing $1201.2 \mathrm{mg} / \mathrm{L} \mathrm{NaCl}, 130.45 \mathrm{mg} / \mathrm{L} \mathrm{CaCl}_{2}, 24.54 \mathrm{mg} / \mathrm{L} \mathrm{MgCl}_{2}$, $3775 \mathrm{mg} / \mathrm{L} \mathrm{KCl}, 137.7 \mathrm{mg} / \mathrm{L} \mathrm{Na}_{2} \mathrm{SO}_{4}, 81.58 \mathrm{mg} / \mathrm{L} \mathrm{Na}_{2} \mathrm{CO}_{3}$, $1163.9 \mathrm{mg} / \mathrm{L} \mathrm{NaHCO}_{3}$. After it was sheared $20 \mathrm{~s}$ in order to simulate the process of site application, the solutions were placed under the temperature $\left(60^{\circ} \mathrm{C}\right)$ for simulating the temperature of oil layer and carried on the aging experiment and then the aging experiment was carried out. The viscosity of the solutions was measured with Brookfield DV-II viscometer and the shear rate was $10 \mathrm{~s}^{-1}$. The relative viscosity for each sample solution was calculated by using the following equation:

$$
\text { Relative viscosity }=\frac{\eta_{\mathrm{t}}}{\eta_{0}} \times 100 \%
$$

wher $\eta_{\mathrm{t}}$ is the apparent viscosity of each degradation sample solution ( $\mathrm{mPa} \mathrm{s}$ ) at time $\mathrm{t}, \eta_{\mathrm{o}}$ is the apparent viscosity of its non-degradation aqueous sample solution ( $\mathrm{mPa} \mathrm{s}$ ).

\section{RESULTS AND DISCUSSION}

Synthesis and compositional analyses: A series of PADO copolymers with twin-tailed structure have been synthesized via the micellar copolymerization procedure using the feed ratios outlined in Table-1. The copolymers contain sulfobetaine (DMAPS) $c a .5 \mathrm{~mol} \%$ incorporation and the content of the hydrophobic monomer (DOMAM) was in the range of 0.1$1.5 \mathrm{~mol} \%$. The conversion of the copolymer decreased with the increase of DOMAM in the feed ratio of monomers. The actual mole ratio of AM/DMAPS/DOMAM in PADO were also calculated according to the elemental analysis and the results were shown in Table-1. Candau and Selb ${ }^{16}$ reported that the high concentration of hydrophobic monomers in the micelles favours their incorporation as blocks in the backbone of hydrophobically modified polyacrylamide and their solution properties are strongly dependent on the hydrophobe/surfactant ratio during the synthesis. Hence, the sequence distribution of the hydrophobic monomers DOMAM in PADO was controlled by varying the number of hydrophobes per micelle, $\mathrm{N}_{\mathrm{H}}$. The key-parameter $\mathrm{N}_{\mathrm{H}}$ is calculated from the following equation:

$$
\mathrm{N}_{\mathrm{H}}=\frac{\left([\mathrm{DOMAM}] \times \mathrm{N}_{\mathrm{agg}}\right)}{([\mathrm{SDS}]-\mathrm{cmc})}
$$

where [DOMAM] is the initial molar concentration of hydrophobic twin-tailed monomer, [SDS] is the molar surfactant concentration, cmc is its critical micellar concentration and $\mathrm{N}_{\text {agg }}$ is its aggregation number. For this system, the $\mathrm{N}_{\text {agg }}$ of SDS is ca. 62 and the $\mathrm{cmc}$ of SDS (at $50^{\circ} \mathrm{C}$ ) was taken to be $9.2 \times$ $10^{-3} \mathrm{~mol} \mathrm{dm}^{-3} 17,19$. The $\mathrm{N}_{\mathrm{H}}$ of the samples was calculated according to the DDMVBA content determined by elemental analysis and the results were shown in Table-2.

${ }^{1}$ H NMR spectra: ${ }^{1} \mathrm{H}$ NMR spectra of DOMAM and PADO-1 as typical cases was shown in Fig. 2. Fig. 2(A) shows that no peaks corresponding to $-\mathrm{C}=\mathrm{CH}_{2}$ are observed in the ${ }^{1} \mathrm{H}$ NMR spectrum. Upon formation of PADO (i.e. PADO-1), new broad signals appear at 1.10-1.15 ppm (peak a) which correspond to the protons of methylene groups in the twin-tailed chain from DOMAM units. At the same time, peak b $(0.88$ ppm) also belongs to the protons of terminal methyl groups for twin-tailed chain. All of them show characteristic peaks due to the protons of PADO-1(A) and DOMAM monomers (B) in Fig. 2, indicating the linking of DMAPS and DOMAM monomers to the polymer backbone. However, it does not give quantitative information on the level of DOMAM incorporation due to the weak signal strength of the protons of hydrophobic units in the spectrum.

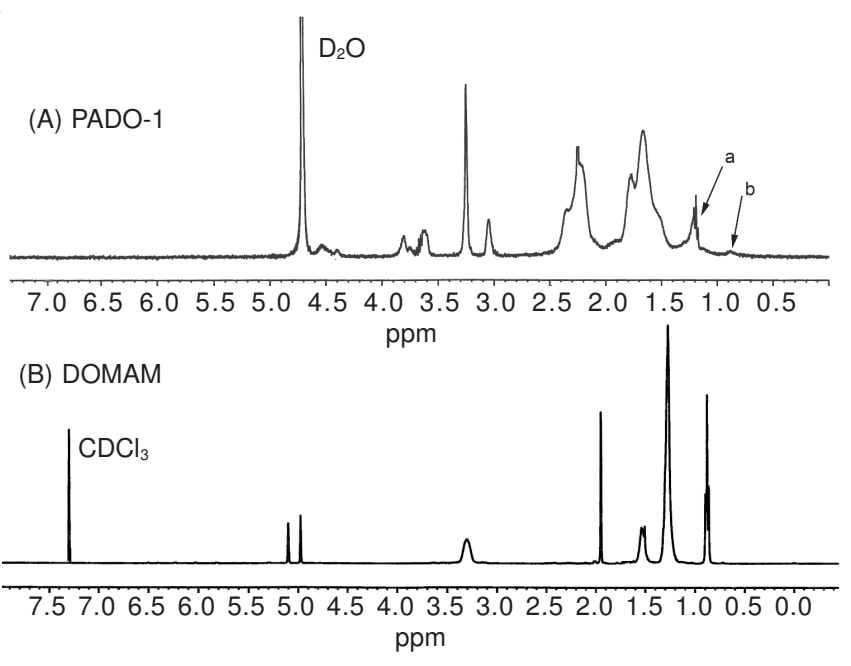

Fig. 2. ${ }^{1} \mathrm{H}$ NMR spectra of PADO-1(A) and twin-tailed monomer DOMAM (B)

Static light scattering (SLS) Measurements: The weight-average molecular weight $\left(\mathrm{M}_{\mathrm{w}}\right)$ of each PADO sample was obtained by SLS analysis. Static light scattering were performed in $0.15 \mathrm{~mol} \mathrm{~L}^{-1} \mathrm{NaCl}$ solution, which is the suitable solvent for molecular mass measurements of hydrophobically modified acrylamide copolymers. In static light scattering, we

\begin{tabular}{|c|c|c|c|c|c|}
\hline \multicolumn{6}{|c|}{$\begin{array}{l}\text { TABLE- } 2 \\
\text { KEY-PARAMETER N } N_{\mathrm{H}} \text {, MOLECULAR WEIGHT, CONSTANT VALUES OF } \mathrm{I}_{1} / \mathrm{I}_{3} \text {, } \\
\text { CMC, VISCOUS ACTIVATION ENERGY AND CONVERSION RATE OF PADO }\end{array}$} \\
\hline Sample & $\mathrm{N}_{\mathrm{H}}{ }^{\mathrm{a}}$ & $10^{-6} \mathrm{Mw}\left(\mathrm{g} \mathrm{mol}^{-1}\right)^{\mathrm{b}}$ & Constant values of $\mathrm{I}_{1} / \mathrm{I}_{3}{ }^{\mathrm{c}}$ & $\mathrm{cmc}(\mathrm{g} / \mathrm{L})$ & Viscous activation energy $\left(\mathrm{KJ} \mathrm{mol}^{-1}\right)$ \\
\hline PADO-1 & 2.38 & 1.13 & 1.21 & $0.0333^{\mathrm{c}} 0.0351^{\mathrm{d}}$ & 8.984 \\
\hline PADO-2 & 6.93 & 1.24 & 1.16 & $0.0187^{\mathrm{c}} 0.0162^{\mathrm{d}}$ & 8.324 \\
\hline PADO-3 & 13.21 & 1.55 & 1.14 & $0.0138^{\mathrm{c}} 0.0122^{\mathrm{d}}$ & 7.321 \\
\hline PADO-4 & 21.23 & 1.65 & 1.01 & $0.0062^{\mathrm{c}} 0.0057^{\mathrm{d}}$ & 6.305 \\
\hline
\end{tabular}
are able to obtain the weight-average molar mass $\mathbf{M}_{w}$, the

${ }^{a}$ The number of hydrophobic monomers per micelle, calculated according to the DOMAM content determined by elemental analysis. ${ }^{b}$ Determined by static light scattering. ${ }^{~}$ Determined by fluorescence spectra. ${ }^{\mathrm{d}}$ Measured by surface tension. 
second-order virial coefficient $\mathrm{A}_{2}$ and the mean square radius $<\mathrm{r}_{\mathrm{g}}^{2}>$ of polymer chains from the angular dependence of the excess absolute scattering intensity, known as the Rayleigh ratio $R(\theta)$, on the basis of

$$
\frac{\mathrm{K} * \mathrm{C}}{\mathrm{R}(\theta)}=\frac{1}{\mathrm{M}_{\mathrm{w}}}\left(1+\frac{1}{3}<\mathrm{r}_{\mathrm{g}}^{2}>\mathrm{g}^{2}\right)+2 \mathrm{~A}_{2} \mathrm{C}
$$

where $\mathrm{K}^{*}=\frac{4 \pi(\mathrm{dn} / \mathrm{dc})^{2} \mathrm{n}_{0}}{\left(\mathrm{~N}_{\mathrm{A}} \lambda_{0}^{4}\right)}$ and $\mathrm{q}=\left(\frac{4 \pi \mathrm{n}_{0}}{\lambda_{0}}\right) \sin \left(\frac{\theta}{2}\right)$, with $\mathrm{n}_{\mathrm{o}}, \mathrm{dn} / \mathrm{dc}, \lambda_{0}$ and $\theta$ being the solvent refractive index, the specific refractive index increment, the wavelength of the incident light in vacuum and the scattering angle, respectively ${ }^{20,21}$.

Fig. 3 shows a typical static Zimm plot of PADO-2, where the concentrations of copolymer in $0.15 \mathrm{~mol} / \mathrm{L} \mathrm{NaCl}$ solution for Zimm plot were $6.025 \times 10^{-4}, 1.198 \times 10^{-3}, 1.795 \times 10^{-3}$, $2.452 \times 10^{-3}, 3.035 \times 10^{-3}, 3.602 \times 10^{-3}$ and $4.212 \times 10^{-3} \mathrm{~g} / \mathrm{mL}$. Using the projection $\theta=0$ and $C=0$, the values of $M_{w}$ could be obtained. The results of $\mathrm{M}_{\mathrm{w}}$ are listed in Table- 1 . However, it shoud be noted that the weight-average molecular weight $\left(\mathrm{M}_{\mathrm{w}}\right)$ of PADO by static light scattering really represents a challenge for the normal solvents used for hydrophobically modified acrylamide copolymers since the hydrophobic twintailed groups induce aggregations, which often leads to more or less erroneous results ${ }^{22}$.

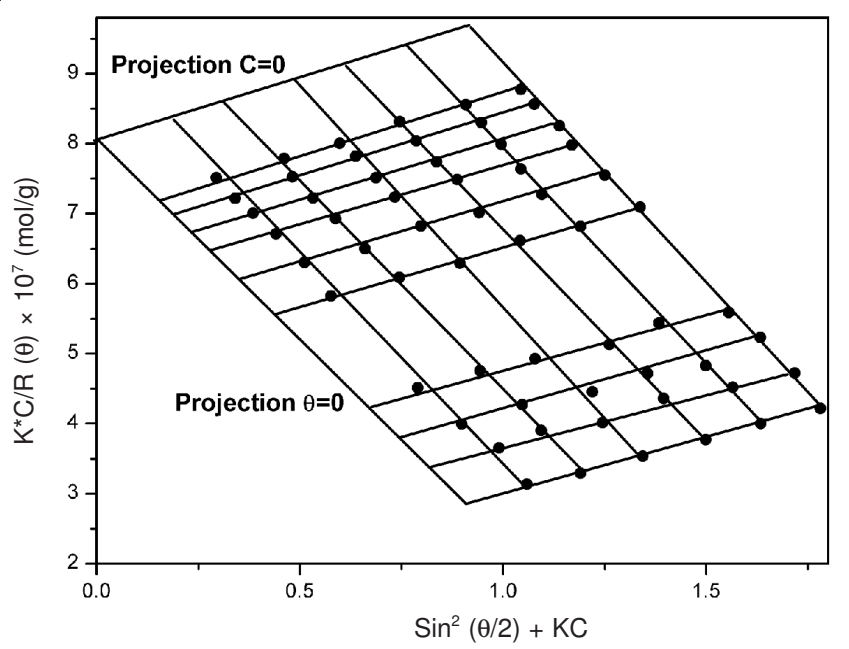

Fig. 3. Typical Zimm plot for PADO-2 in $0.15 \mathrm{~mol} \mathrm{~L}^{-1} \mathrm{NaCl}$ solution

Thermal properties of PADO: Fig. 4 was the TGA and DTG curves of PADO-1 to 4 (the curves of a-d). The copolymers showed two major weight loss steps under nitrogen atmosphere. The first transition occurred around $290^{\circ} \mathrm{C}$, which might be associated with the loss of side groups. From the DTG curves, it can be seen that the decomposition temperature increased with the decreasing mole ratio of DMAPS/DOMAM in copolymer. This deviation is due to the fact that bulky steric hindrance of DOMAM damages the hydrogen bond of the amide groups, leading to the decrease of decomposition temperature of the side groups. The second transition occurred at $c a .385^{\circ} \mathrm{C}$ and was associated with the decomposition of the copolymer backbone and the decomposition temperature decreased slightly with the increasing mole percentage
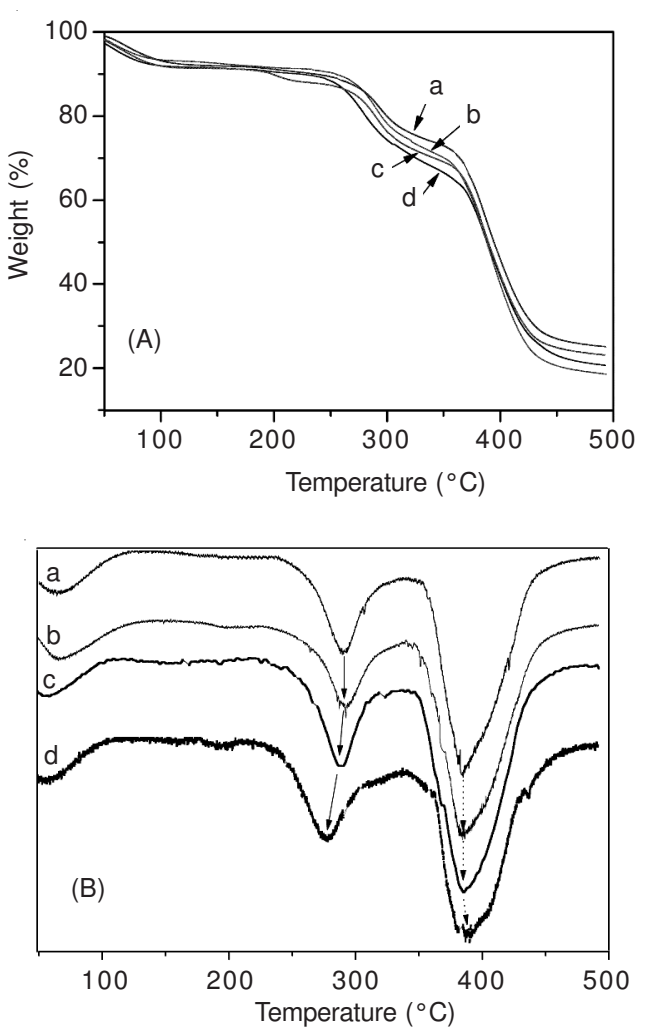

Fig. 4. TGA curves (A) and DTG curves (B) of PADO-1 (a), PADO-2 (b), PADO-3 (c) and PADO-4 (d)

of DOMAM, which might be attributed to the decreasing molecular weight of PADO.

Dynamic light scattering (DLS) measurements: The distribution of the hydrodynamic radius of PADO-3 in $0.1 \mathrm{~mol} /$ $\mathrm{L} \mathrm{NaCl}$ solution obtained by DLS was shown in Fig. 5. It can be seen that a peak corresponding to the hydrodynamic radius may be due to the aggregates in the solution. It shows clearly that the size of aggregates increased with increasing copolymer concentration. The size distribution is broad at high concentrations level. The broad size distribution at high copolymer concentration level is due to the coexistence of individual copolymer chains and different size clusters in $0.1 \mathrm{~mol} / \mathrm{L} \mathrm{NaCl}$ solutions. The distribution of the hydrodynamic radius increases and broadens with increasing the concentration of PADO-3 from 5 to $100 \mathrm{mg} / \mathrm{L}$ due to intramolecular and the intermolecular interaction of sample containing hydrophobic twin-tailed chain, which indicates the dependence of the aggregates on the molecular association of hydrophobic groups in the solution. The competition between intramolecular and the intermolecular interaction controls the sizes of the aggregates in the solution which would affect the overall solution behaviour ${ }^{23-25}$.

The effects of $\mathrm{NaCl}$ concentration on the average hydrodynamic radius $(\mathrm{Rh})$ for PADO were shown in Fig. 6. The Rh of all the copolymer samples increases as a consequence of increased intermolecular association with the increase of the concentration of $\mathrm{NaCl}$, which correlates well with the known effects of $\mathrm{NaCl}$ on the hydrophobic and zwitter ionic associations. When even a few of $\mathrm{NaCl}$ was added to the copolymer solution $(0.05 \mathrm{~mol} / \mathrm{L})$, the hydrodynamic radius increased obviously. It suggests that the appearance of $\mathrm{NaCl}$ had great 


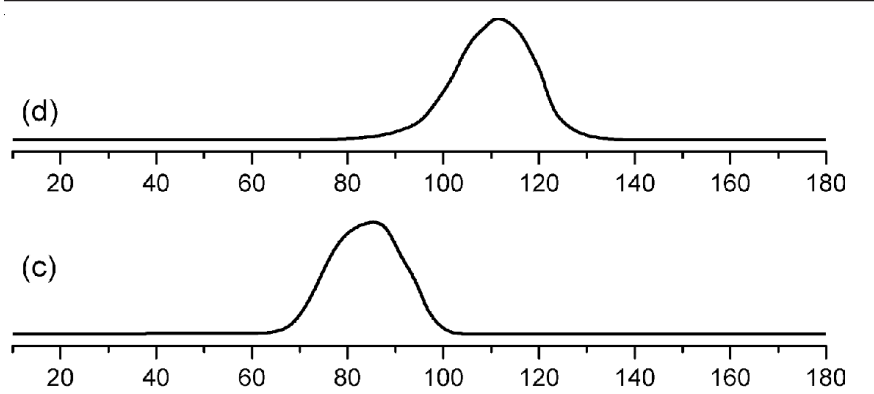

(b)
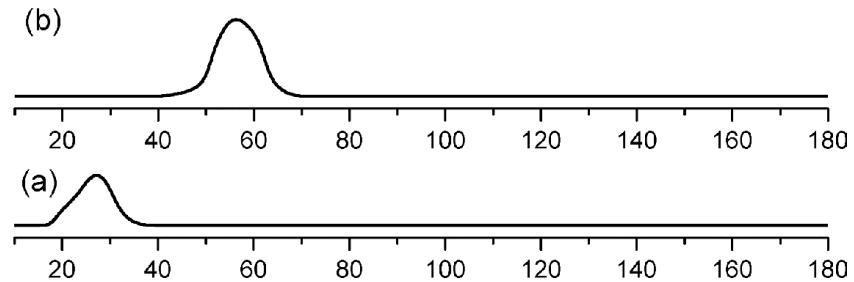

Hydrodynamic diameters $(\mathrm{nm})$

Fig. 5. Size distribution of the aggregates formed by PADO-3 in $0.1 \mathrm{~mol} /$ $\mathrm{L} \mathrm{NaCl}$ solutions at the concentrations of (a) $5 \mathrm{mg} / \mathrm{L}$, (b) $50 \mathrm{mg} / \mathrm{L}$, (c) $75 \mathrm{mg} / \mathrm{L}$ and (d) $100 \mathrm{mg} / \mathrm{L}$ determined by DLS at $25^{\circ} \mathrm{C}$

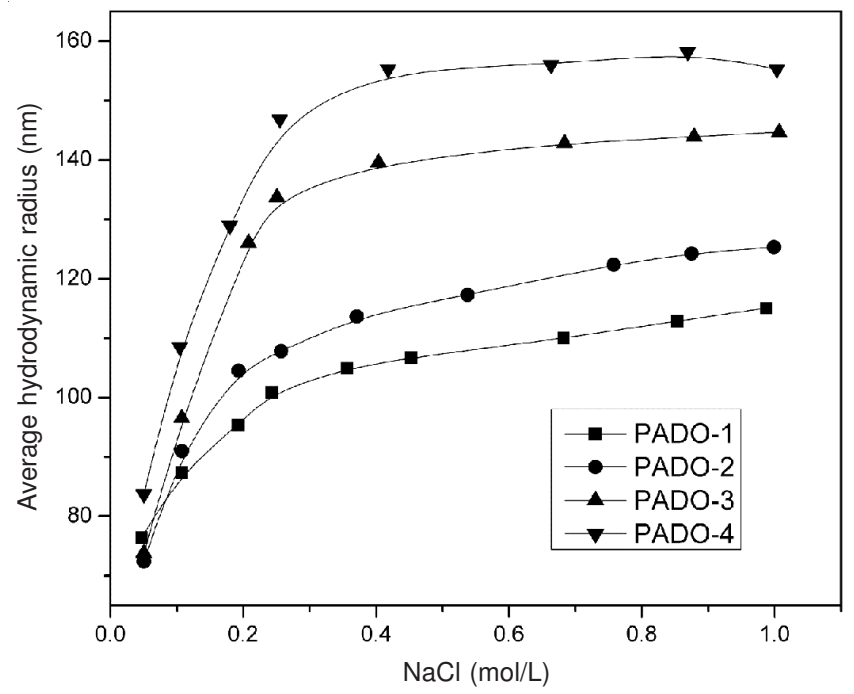

Fig. 6. Variation of the average hydrodynamic radius $(\mathrm{Rh})$ with salt concentration at $25^{\circ} \mathrm{C}\left(\mathrm{C}_{\mathrm{PADO}}=75 \mathrm{mg} / \mathrm{L}\right)$

influence to the aggregation of PADO in the solution. When the $\mathrm{NaCl}$ concentration increased to $0.25 \mathrm{~mol} / \mathrm{L}$, the hydrodynamic radius had further increase. But the increase was not remarkable when the concentration increased from 0.25 to 1.00 $\mathrm{mol} / \mathrm{L}$. These results indicate that the incorporation of the zwitter ionic groups promotes hydrophobic intermolecular association in the presence of added salt and the hydrophobic intermolecular association is enhanced by increasing the length of the hydrophobic block length ${ }^{26-27}$.

Fluorescence spectra: The fluorescence of pyrene is known to be sensitive to changes in the microenvironment. In emission spectra, the relative intensities of the first vibronic peak $\left(I_{1}\right)$ and the third vibronic peak $\left(I_{3}\right)$ were sensitive to the environment polarity and are frequently used for the determination of aggregate polarity. The intensity ratio of the first to the third peaks, $\mathrm{I}_{1} / \mathrm{I}_{3}$, is often used as a measure of the polarity of the microenvironment $t^{30-35}$. For the hydrophobically modified copolymers, there is a certain concentration at which the interpolymer association starts to occur abruptly. Then the critical polymer concentration is defined as the $\mathrm{cmc}$ for aggregate-forming polymers. When PADO self-assembly forms, pyrene molecules can get into the interior hydrophobic domain of PADO micelles in the solution, thus this will result in an abrupt change of the $\mathrm{I}_{1} / \mathrm{I}_{3}$ ratio. The fluorescence spectra using the pyrene probe of PADO at $25^{\circ} \mathrm{C}$ are shown in Fig. 7, from which it can be seen that, in the range of low concentration, the $I_{1} / I_{3}$ ratios for PADO decrease slowly with the concentration of PADO increase, which can be attributed to the fact that a large fraction of the pyrene molecules found hydrophobic sites and only few pyrene molecules distributed into a nonaqueous environment because there aren't more copolymers of PADO. As the concentration of PADO is increased, the $I_{1} / I_{3}$ ratios decrease significantly at a certain concentration, indicating the onset of intermolecular association. At high concentration of PADO, the $\mathrm{I}_{1} / \mathrm{I}_{3}$ ratios of these polyelectrolytes reach a constant value and the constant values of $\mathrm{I}_{1} / \mathrm{I}_{3}$ for PADO follows a sequence, i.e., $\mathrm{PADO}-1>\mathrm{PADO}-$ $2>$ PADO-3 $>$ PADO- 4 . The results indicates that the content of the double hydrophobic chains in PADO can result in synergic hydrophobic effects and cause a high intensity of hydrophobic interaction, which plays a key role in reducing the constant values of $I_{1} / I_{3}$.

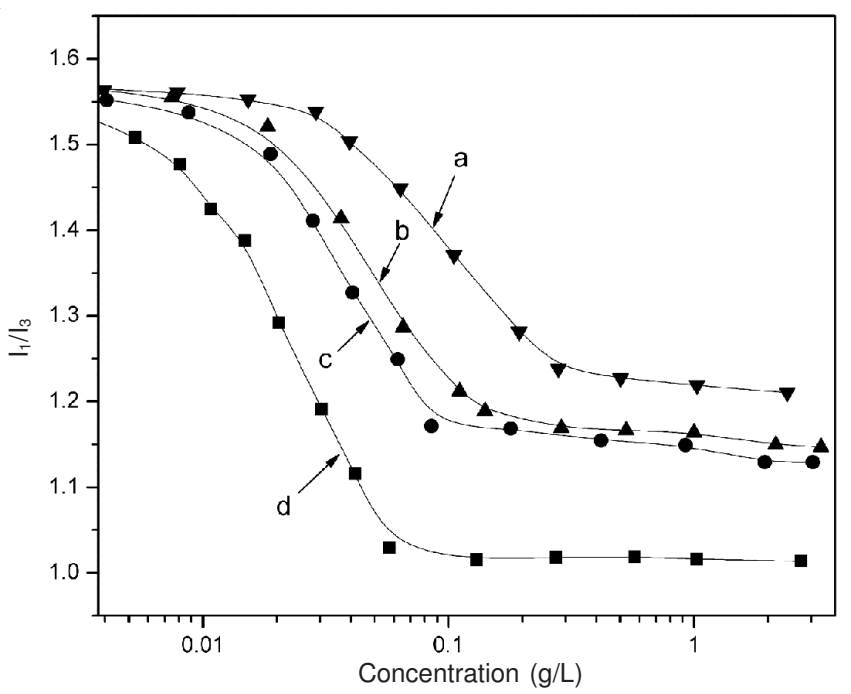

Fig. 7. $I_{1} / I_{3}$ ratio of pyrene as a function of concentration for PADO: PADO1(a), PADO-2(b), PADO-3(c) and PADO-4 (d)

Surface tension: Fig. 8 showed the relationship between surface tension and concentration of PADO samples at $25^{\circ} \mathrm{C}$. It is clear that the relationship between surface tension and concentration of PADO is characterized by two distinguishable regions, one at the lower concentration range characterized by a fast decrease in the surface tension values, the other at the higher concentrations, in which the surface tension values remain almost constant. The concentration at the break point that separates the two regions is regarded as the critical micelle concentration $(\mathrm{cmc})$. The values of $\mathrm{cmc}$ for PADO are listed in Table-2. For solutions of PADO, the cmc corresponding to the onset of decrease in surface tension corresponds closely to the onset of association concentration determined by fluorescence spectra. All of that indicate the hydrophobic 


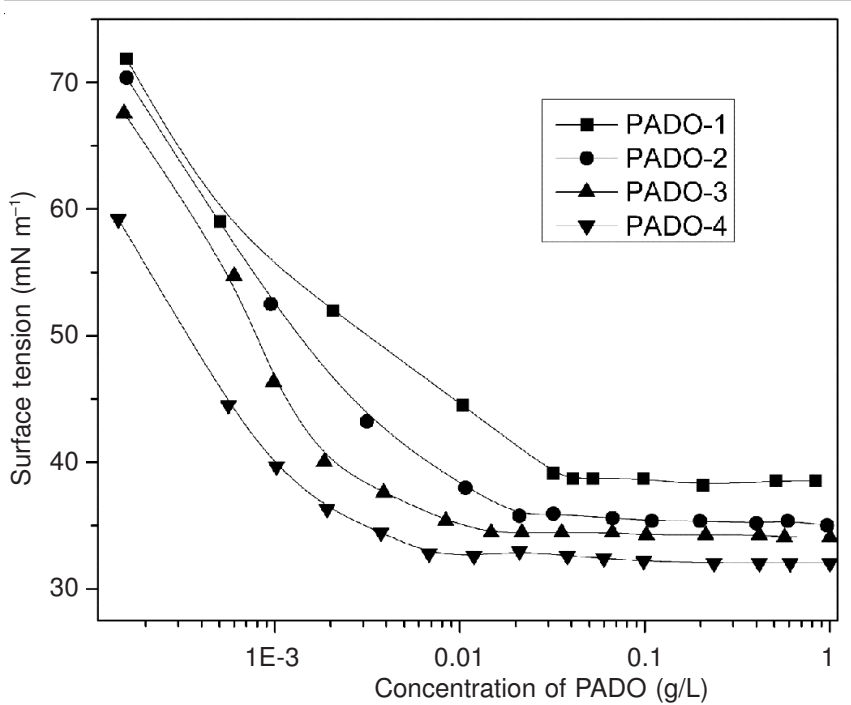

Fig. 8. Changes in the surface tension of PADO solutions as a function of PADO concentration at $25^{\circ} \mathrm{C}$

twin-tailed block of PADO induce the screening of the electrostatic repulsion among the polar head groups and leads to remarkably lower cmc and surface tension in comparison.

Thermal degradation of PADO samples in brine solutions: Fig. 9 shows that the viscosities of PADO in 0.2 $\mathrm{mol} / \mathrm{L} \mathrm{NaCl}$ solutions decrease as temperature increases, which indicates that the increasing of temperature can give rise to crimple of PADO molecules due to its dehydrating and destruction of the associational structure. As well known, the relationship between apparent viscosities of polymer solution and temperatures satisfies Arrhenius equation:

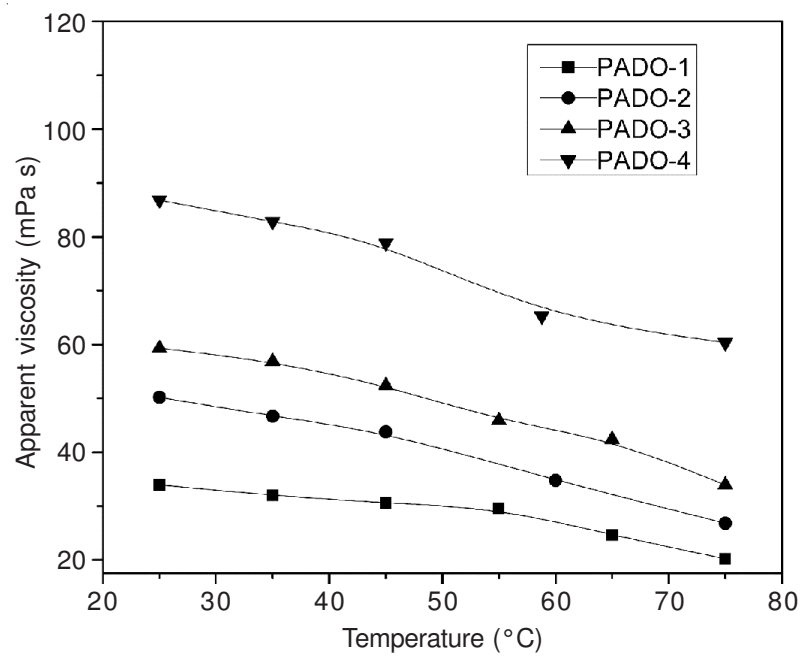

Fig. 9. Viscosity of PADO $\left(\mathrm{Cp}=0.3 \mathrm{wt}\right.$. \%) at $10 \mathrm{~s}^{-1}$ as function of temperature in $0.2 \mathrm{~mol} / \mathrm{L} \mathrm{NaCl}$ solutions

$$
\eta=A \exp \left(\frac{\Delta \mathrm{E} \eta}{\mathrm{RT}}\right)
$$

where $\eta$ is the apparent viscosity of copolymer solution, $\Delta \mathrm{E}_{\eta}$ is the viscous activation energy; $\mathrm{R}$ is the gas constant and $\mathrm{T}$ is the absolute temperature. According to this eqn. $3, \ln \eta$ and the reciprocal of temperature of the polymer solution shows a straight-line relationship in Fig. 9, with slope of $\Delta E \eta / R$. As a consequence, viscous activation energy $\triangle \mathrm{E} \eta$ of PADO in 0.2 $\mathrm{mol} / \mathrm{L} \mathrm{NaCl}$ solutions was calculated (Table-2). The results show that both the viscous activation energy of PADO and the apparent viscosities of PADO as a function of temperature decrease slightly with the increasing in the content of the double hydrophobic chains in PADO, which indicated that PADO system containing twin-tailed hydrophobic groups has better performance on the temperature resistance.

The relative viscosity as a function of degradation time for PADO series in the simulated water at $60{ }^{\circ} \mathrm{C}$ is showed in Fig. 10. As we can see from Fig. 10, the relative viscosity was $43.35 \%$ for PADO-4 sample and that for PADO-1 was $34.69 \%$ after degradation for $240 \mathrm{~h}$ in the simulated water. Moreover, it was in the order of PADO-4 > PADO-3 > PADO-2 > PADO-1 for the relative viscosity in the simulated water after $100 \mathrm{~h}$. It was consistent with the order of twin-tailed group content in the PADO. This may indicate that hydrodynamic twin-tailed blocks play an important role in salt tolerance and temperature resistance.

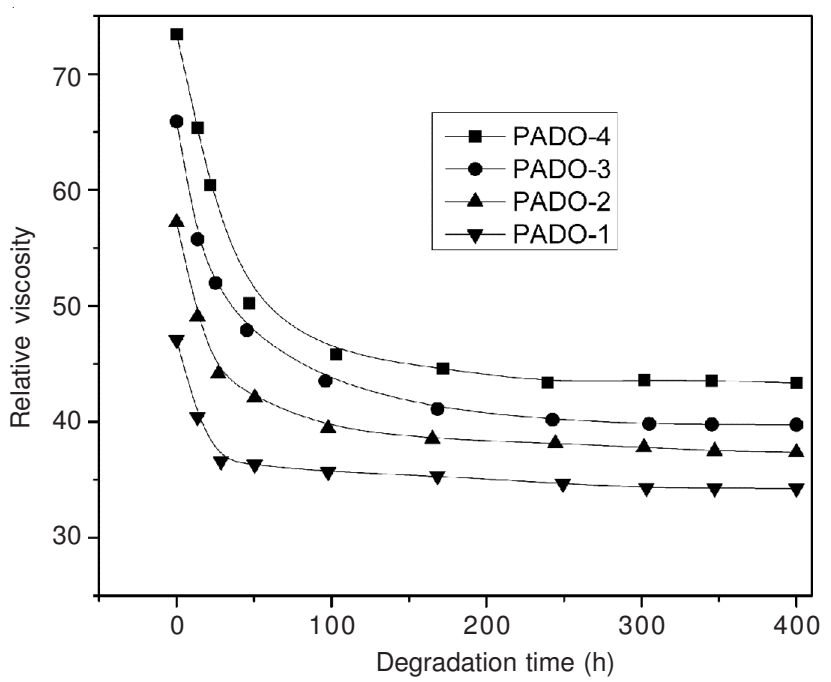

Fig. 10. Variation of relative viscosity as a function of degradation time for PADO series $\left(0.3\right.$ wt. \%) in the simulated water at $60{ }^{\circ} \mathrm{C}$

In the simulated water, the high ionic strength can suppress the formation of inner salt for PADO molecules and be in favour of the extension of molecule chains. Moreover, it helps the association of hydrophobic twin-tailed parts of PADO. The presence of inorganic electrolytes also enhances the electrostatic interaction of PADO, resulting in the increase of hydrodynamic volume and even forming a spatial network structure, so that it can increase the viscosity of the solution ${ }^{36}$. In a word, PADO forms interaction through the static electricity force gathering electrolyte compound and increases the molecular hydrodynamic volume, so that it has also higher viscosity values and enhances the salt resistance and thermal stability. In addition, the experimental conditions are simulated for oil well environment, this experiment might provide information for the improvement of PADO series used in polymer flooding.

\section{Conclusion}

Novel twin-tailed associative copolymers PADO were prepared by using a micellar copolymerization route and the 
molecular weights of PADO series were ascertained from 1.13 $\times 10^{6}$ to $1.65 \times 10^{6} \mathrm{~g} / \mathrm{mol}$ by SLS. Then the copolymers PADO were characterized extensively via ${ }^{1} \mathrm{H}$ NMR, EA and TGA. The results showed that the bulky steric hindrance of DOMAM can damage the hydrogen bond of the amide groups, leading to the decrease of decomposition temperature of side groups. Surface activity and aggregation properties of PADO series were studied by DLS, surface tension and fluorescence measurements. The cmc values of PADO series measured by fluorescence measurements and identify nearly with that obtained by surface tension measurements. The results from DLS indicate that the distribution of Rh increases and broadens with increasing the content of twin-tailed hydrophobic parts in PADO due to intramolecular and the intermolecular interaction of sample. The viscosity stability results also suggest that PADO can greatly improve salt resistance and thermal stability and it has the high practical application performance in oil recovery.

\section{ACKNOWLEDGEMENTS}

The authors gratefully acknowledged the financial support from Major Research of Science and Technology, China (Grant No. 2011ZX05024-004-08) and National Basic Research Program of China (973 Program, 2009CB930103).

\section{REFERENCES}

1. W.B. Li, H.Y. An, Y. Tan, C. Lu, C. Liu, P. Li, K. Xu and P. Wang, Soft Matter, 8, 5078 (2012).

2. H.J. Ren, W. Chen, Y.F. Zheng and Z.K. Luan, React. Funct Polym., 67, 601 (2007).

3. E. Antunes, F. Garcia, P. Ferreira, A. Blanco, C. Negro and M.G. Rasteiro, Ind. Eng. Chem. Res., 47, 9370 (2008).

4. S. Evani and G.D. Rose, Polym. Mater. Sc Eng., 57, 471 (1987).

5. M. Rodriguez, J. Xue and L.M. Gouveia, Colloid Surf. A, 373, 66 (2011).

6. I.V. Blagodatskikh, O.V. Vasileva, E.M. Ivanova, S.V. Bykov, N.A. Churochkina, T.A. Pryakhina, V.A. Smirnov, O.E. Philippova and A.R. Khokhlov, Polymer, 45, 5897 (2004).
7. E.J. Jimenez-Regalado, G. Cadenas-Pliego, M. Pérez-Álvarez and Y. Hernández-Valdez, Polymer, 45, 1993 (2004).

8. W. Xue and I.W. Hamley, Polymer, 43, 3069 (2002)

9. E. Volpert, J. Selb and F. Candau, Macromolecules, 29, 1452 (1996).

10. D.J. Liaw, W.F. Lee, Y.C. Whung and M.C. Lin, J. Appl. Polym. Sci., 34, 999 (1987)

11. Y.J. Che, Y.B. Tan, J. Cao, H.P. Xin and G.Y. Xu, Polym. Bull., 66, 17 (2011).

12. C.L. McCormick, T. Nonaka and C.B. Johnson, Polymer, 29, 731 (1988).

13. D.E. Bergbreiter, N.A. Avilés-Ramos and D. Ortiz-Acosta, J. Comb. Chem., 9, 609 (2007).

14. W. Wolfgang, W. Klaus, B. Artur and R. Frank, De Pat 4341986 (1995).

15. A. Hill, F. Candau and J. Selb, Macromolecules, 26, 4521 (1993).

16. F. Candau and J. Selb, Adv. Colloid Interf., 79, 149 (1999).

17. K.M. Johnson, M.J. Fevola and C.L. McCormick, J. Appl. Polym. Sci., 92, 647 (2004).

18. Y.J. Che, Y.B. Tan, J. Cao and G.-Y. Xu, J. Polym. Res., 17, 557 (2010).

19. K.D. Branham, D.L. Davis, J.C. Middleton and C.L. McCormick, Polymer, 35, 4429 (1994).

20. P. Debye, J. Phys. Colloid Chem., 51, 18 (1947).

21. B.H. Zimm, J. Chem. Phys., 16, 1099 (1948).

22. Y.J. Feng, L. Billon, B. Grassl, G. Bastiat, O. Borisov and J. François, Polymer, 46, 9283 (2005).

23. X.L. Huang, Y.B. Tan, Q.F. Zhou, Y.X. Wang and Y.J. Che, Carbohydr. Polym., 74, 685 (2008).

24. Y.J. Che, Y.B. Tan, X.N. Ren, H.P. Xin and F.J. Meng, Colloid Polym. Sci., 290, 1237 (2012).

25. J.L. Wu, Y.M. Wang, M. Hara, M. Granville and R.J. Jerome, Macromolecules, 27, 1195 (1994).

26. M.S. Mozumder, R.S. Alnaizy, Y. Umar, S.A. Ali and B.F. Abu-Sharkh, Eur. Polym. J., 41, 2224 (2005).

27. C.L. McCormick and L.C. Salazar, Polymer, 33, 4617 (1992).

28. E.E. Kathmann, L.A. White and C.L. McCormick, Polymer, 38, 871 (1997).

29. B. Gao, L. Jiang and K. Liu, Eur. Polym. J., 43, 4530 (2007).

30. M. Wilhelm, C.L. Zhao, Y. Wang, R. Xu, M.A. Winnik, J.L. Mura, G. Riess and M.D. Croucher, Macromolecules, 24, 1033 (1991).

31. I. Astafieva, X.F. Zhong and A. Eisenberg, Macromolecules, 26, 7339 (1993).

32. R. Zana, M. In, H. Lévy and G. Duportail, Langmuir, 13, 5552 (1997).

33. P.K. Weissenborn and R.J. Pugh, J. Colloid Interf. Sci., 184, 550 (1996).

34. N.J. Turro and A. Yekta, J. Am. Chem. Soc., 100, 5951 (1978).

35. M. Koyanagi, J. Mol. Spectrosc., 25, 273 (1968).

36. L. Zhang, X. Lv, Y.J. Zhu, J. Zhang, H. Wang and Y.B. Tan, Colloid Polym. Sci., 289, 1579 (2011). 